\title{
CURVATURE OF NONLINEAR CONNECTIONS
}

\author{
JAAK VILMS
}

1. Introduction. The curvature of a homogeneous (nonlinear) connection on a vector bundle (and in particular, on the tangent bundle) has been defined by several authors in terms of local reference frames. (See [1] and the references listed there.)

In this note, an intrinsic definition is given for the curvature of a general nonlinear connection on a smooth $\left(C^{\infty}\right)$ vector bundle (modeled on a Banach space [3]).

Let $p: E \rightarrow X$ be a smooth vector bundle over a smooth manifold $X$. A (general nonlinear) smooth connection on it [5] is a smooth splitting of the (direct) exact sequence

$$
0 \rightarrow V E \stackrel{J}{\rightarrow} T E \stackrel{p^{\prime}}{\rightarrow} p^{-1} T X \rightarrow 0
$$

of vector bundles over the manifold $E$ (where $p^{-1} T Y$ denotes the pullback, $p^{\prime}$ is defined by factoring the tangent map $p_{*}: T E \rightarrow T X$ through $p^{-1} T X$, and $V E$ is the kernel of $p^{\prime}$ (or of $p_{*}$ ) with $J$ its inclusion).

The splitting is given by a smooth morphism $V: T E \rightarrow V E$ such that $V J=I$, or equivalently by a smooth morphism $W: p^{-1} T X \rightarrow T E$ such that $p^{\prime} W=I . V$ and $W$ (the left and right splitting maps, respectively) are related by $J V+W p^{\prime}=I$. In other words, $V=J V$ and $H=W p^{\prime}$ are the projection maps of a direct sum decomposition $T E=H E \oplus V E$ (where $H E=$ kernel $W=$ image $V$ ). Of course $H E$, the horizontal bundle, is isomorphic to $p^{-1} T X$.

On the other hand, the vertical bundle, $V E$, is canonically isomorphic to $p^{-1} E$. Hence there is a canonical map $r: V E \rightarrow E$ over $p$ (isomorphic on the fibres). $D=r V: T E \rightarrow E$ is the connection map. It is a smooth morphism of the tangent bundle structure on $T E$, and is also fibre-preserving for the fibres of the other fibre bundle structure, $p_{*}: T E \rightarrow T X$, on $T E$. If $D$ is linear on the $p_{*}$ fibres, one has a linear connection; if $D$ is merely 1 -homogeneous, then a homogeneous connection (also called a nonlinear connection by most authors).

REMARK. If $E_{0} \subset E$ is an open set, a smooth splitting of (1) restricted over $E_{0}$ is a smooth connection on $E_{0}$. This added generality is needed for strictly nonlinear connections. Namely, a smooth homogeneous connection is always assumed to be on $E_{0}=E-0$ (because one on $E$ is necessarily linear).

Received by the editors May 11, 1967. 
The left splitting map $V$ is a vertical bundle valued 1 -form on the manifold $E$; hence $V$ is the connection form. The curvature form is its exterior derivative, $d V$, taken with respect to the linear Berwald connection on the vertical bundle, which will be shown to exist next.

2. The Berwald connection. Let $U$ be the domain of a chart on $X$ (and identify it with its homeomorphic image in the model space $B$ of $X$ ); let $T X \mid U \approx U \times B$ be the tangent chart. Suppose $E \mid U$ $\approx U \times E$ is a chart on $E$; by taking tangent maps one gets a chart $T E \mid(E \mid U) \approx U \times E \times B \times E$. The sequence (1) over $E \mid U$ becomes the sequence

$$
0 \rightarrow U \times E \times 0 \times E \rightarrow U \times E \times B \times E \rightarrow U \times E \times B \rightarrow 0
$$

of bundles over $U \times E$, with $p^{\prime}(x, a, \lambda, b)=(x, a, \lambda)$. The map $r: V E \rightarrow E$ is locally $r(x, a, 0, b)=(x, b)$ and $V(x, a, \lambda, b)=(x, a, 0, b+\omega(x, a) \lambda)$, whence $D(x, a, \lambda, b)=(x, b+\omega(x, a) \lambda)$.

Here the smooth map $\omega: U \times E \rightarrow L(B, E)$ is the local component of the connection for this chart [5]. The connection is linear or homogeneous iff each $\omega$ is, in its second variable, $a$. In the linear case $\Gamma(x)(a, \lambda)=\omega(x, a) \lambda$ defines a smooth map $\Gamma: U \rightarrow L^{2}(E, B ; E)$, the local Christoffel component.

Note if the connection is on $E_{0} \subset E$, $\omega$ is defined on $U \times E_{U} \subset U \times E$; e.g. $E_{U}=E-0$ in the homogeneous case. Let $\partial_{i}$ denote the $i$ th partial (Fréchet) derivative (written as $D_{i}$ in [3, Chapter 1]).

Proposition. For a smooth connection on $E_{0} \subset E$, the maps $\Omega:\left(U \times E_{U}\right) \times E \rightarrow L(B \times E, E)$ defined by $\Omega((x, a), b)(\mu, c)=\partial_{2} \omega(x, a)(b) \mu$ are the local components of a linear connection on $V E \mid E_{0} \rightarrow E_{0}$ (the Berwald connection).

Proof. If $P(x, a)=(f x, A(x) a)$ is a change of charts $U \times E \approx U \times E$ on $E$, the corresponding change of charts $P_{*}$ on $T E$ is given by

$$
P_{*}(x, a, \lambda, b)=\left(f x, A(x) a, f^{\prime}(x) \lambda, A^{\prime}(x)(\lambda) a+A(x) b\right)
$$

with primes denoting (Fréchet) derivatives. The local equation for $D$ shows that the old and new local components $\omega$ and $\bar{\omega}$ are related by the classical equation

$$
A^{\prime}(x)(\lambda) a+\bar{\omega}(f x, A(x) a) f^{\prime}(x) \lambda=A(x) \omega(x, a) \lambda .
$$

Differentiating partially with respect to $a$ in direction $b$ and setting $\lambda=\mu$ produces

$$
A^{\prime}(x)(\mu) b+\partial_{2} \bar{\omega}(f x, A(x) a)(A(x) b) f^{\prime}(x) \mu=A(x) \partial_{2} \omega(x, a)(b) \mu .
$$


Since locally $T(V E) \approx(U \times E \times 0 \times E) \times B \times E \times 0 \times E$, a connection map for a connection on $V E \mid E_{0} \rightarrow E_{0}$ is locally $D(x, a, 0, b ; \mu, c, 0, d)$ $=(x, a ; 0, d+\Omega((x, a), b)(\mu, c))$, where $\Omega:\left(U \times E_{U}\right) \times E \rightarrow L(B \times E, E)$ is smooth. The induced change of charts $P_{* *}$ on $T(V E)$ is $P_{* *}(x, a, 0, b ; \mu, c, 0, d)=\left(f x, A(x) a, 0, A(x) b ; f^{\prime}(x) \mu, A^{\prime}(x)(\mu) a\right.$ $\left.+A(x) c, 0, A^{\prime}(x)(\mu) b+A(x) d\right)$. Hence the old and new $\Omega$ and $\bar{\Omega}$ are related by

$$
\begin{array}{r}
A^{\prime}(x)(\mu) b+\vec{\Omega}((f x, A(x) a), A(x) b)\left(f^{\prime}(x) \mu, A^{\prime}(x)(\mu) a+A(x) c\right) \\
=A(x) \Omega((x, a), b)(\mu, c) .
\end{array}
$$

For $\Omega$ defined by $\Omega((x, a), b)(\mu, c)=\partial_{2} \omega(x, a)(b) \mu$, (2) says this equation is satisfied. Note $\Omega$ is linear in its second variable, $b$. q.e.d.

REMARK 1. A conceptual existence proof goes as follows. Let $V_{1}: T(T E) \rightarrow V(T E)$ be the connection form of the induced connection on $p_{*}: T E \rightarrow T X,\left[5\right.$, Theorem 1], where $V(T E)=$ kernel $p_{*}$. Since $V E \subset T E, V_{1}$ restricts to a map $V_{1}: T(V E) \rightarrow V(T E) \mid V E$. Now this latter bundle is canonically isomorphic to the bundle $v_{1}^{-1}\left(v^{-1} V E\right)$, where $v: V E \rightarrow E$ and $v_{1}: v^{-1} V E \rightarrow V E$. But $v^{-1} V E \approx V(V E)=$ kernel $v$. Hence there is a canonical epimorphism $g: V(T E) \mid V E \rightarrow V(V E)$, and $g V_{1}$ is the connection form of a linear connection on $v: V E \rightarrow E$.

REMARK 2. The original Berwald connection occurs in the case $E=T X$ and the connection is the canonical (homogeneous) connection of a smooth Finsler structure on $X$. (See the references in [1].)

3. The curvature form. For a smooth connection on the vector bundle $F \rightarrow X$, the covariant derivative $D_{u} A$ for $A$ a smooth section of $F$ and $u$ a vector field on $X$ is defined to be $D A_{*} u$ [2]. If the connection is linear, the exterior derivative of a smooth $F$-valued $r$-form $M$ on $X$ (i.e. a smooth antisymmetric section of $L^{r}(T X ; F) \rightarrow X$ ) is the smooth $F$-valued $(r+1)$-form $d M$ on $X$ defined by

$$
\begin{aligned}
d M\left(u_{0}, u_{1}, \cdots, u_{r}\right) & =\sum_{i=0}^{r}(-1)^{i} D_{u_{i}} M\left(u_{0}, \cdots, \hat{u}_{i}, \cdots, u_{r}\right) \\
& +\sum_{i<j}(-1)^{i+j} M\left(\left[u_{i}, u_{j}\right], \cdots, \hat{u}_{i}, \cdots, u_{j}, \cdots, u_{r}\right),
\end{aligned}
$$

where $u_{i}$ are smooth vector fields on $X$. (Smoothness of the Christoffel component $\Gamma(x)(-,-)=\omega\left(x,{ }_{-}\right)_{-}$implies $d M$ is a smooth section.)

For the case $r=1$ the above equation reduces to

$$
d M(u, v)=D_{u} M v-D_{v} M u-M[u, v] .
$$

In terms of a local chart, $M$ is represented by a smooth map 


$$
m: U \rightarrow L(B, F),
$$

and $d M$ is represented by the smooth map $U \rightarrow L^{2}(B ; F)$ defined by (4) $d m(x)(\lambda, \mu)=m^{\prime}(x)(\lambda) \mu-m^{\prime}(x)(\mu) \lambda+\omega(x, m(x) \mu) \lambda-\omega(x, m(x) \lambda) \mu$.

Now the connection form $V$ of a smooth connection on $E_{0} \subset E$ is locally the smooth map $U \times E_{U} \rightarrow L(B \times E, E)$ defined by $\left.V(x, a) \lambda, b\right)$ $=b+\omega(x, a) \lambda$. Using the Berwald connection on $V E \mid E_{0} \rightarrow E_{0}$, (4) implies that the curvature form $d V$ is locally

$$
\begin{aligned}
d V(x, a)((\lambda, b),(\mu, c))= & \omega^{\prime}(x, a)(\lambda,-\omega(x, a) \lambda) \mu \\
& -\omega^{\prime}(x, a)(\mu,-\omega(x, a) \mu) \lambda,
\end{aligned}
$$

where $(x, a) \in U \times E_{U}$. Clearly if either of $(\lambda, b)$ or $(\mu, c)$ is vertical, i.e. if $\lambda=0$ or $\mu=0$, then the right side is 0 . Hence

Lemma. The curvature form $d V$ is horizontal, i.e. $d V(A, B)$ $=d V(H A, H B)$ for all $A, B \in T_{e} E, e \in E_{0}$.

Theorem (Structure equation). If $A$ and $B$ denote vector fields on $E_{0}$, then $d V(A, B)=-V[H A, H B]$.

Proof. Immediate from (3) and the lemma.

An immediate consequence of the structure equation is the following generalization of a result of Sasaki for Riemannian connections $[4$, p. 343].

Corollary. $H E \subset T E$ (over $E_{0}$ ) is integrable iff $d V=0$.

4. The curvature tensor field. The smooth isomorphisms $V E \approx p^{-1} E$ and $H E \approx p^{-1} T X$ are fibre-wise given by $r_{e}:(V E)_{e} \approx E_{x}$ and $p_{*}(e):(H E)_{e} \approx T_{x} X$ (for $\left.p e=x\right)$. Their inverses are the vertical and horizontal lift maps [2], denoted $f_{e}^{V}=r_{e}^{-1}(f) \in(V E)_{e}$ and $u_{e}^{H}=p_{*}(e)^{-1}(u)$ $=W_{e}(u) \in(H E)_{e}$, where $f \in E_{x}$ and $u \in T_{x} X$. These maps induce isomorphisms

$$
L^{2}\left((B E)_{e} ;(V E)_{e}\right) \approx L^{2}\left(T_{x} X ; E_{x}\right) \quad \text { for } e \in E_{0} \quad \text { with } p e=x .
$$

Since each $d V(e)$ is horizontal, it corresponds to an antisymmetric map $R_{x}(-,-) e \in L^{2}\left(T_{x} X ; E_{x}\right)$ by the above isomorphism. That is, it is defined as $R_{x}(u, v)=r(e) d V\left(u_{e}^{H}, v_{e}^{B}\right)$, or locally as

$$
\begin{aligned}
R(x)(\lambda, \mu) a= & \text { the right side of }(5), \text { which by }[3, \text { Proposition 11, p. 8] is } \\
= & \partial_{1} \omega(x, a)(\lambda) \mu-\partial_{1} \omega(x, a)(\mu) \lambda \\
& +\partial_{2} \omega(x, a)(\omega(x, a) \mu) \lambda-\partial_{2} \omega(x, a)(\omega(x, a) \lambda) \mu .
\end{aligned}
$$

(This is equation (28) of $\left[1\right.$, p. 138], with $\theta^{h}=d x^{h}$.) 
The totality of maps $R_{x}(-,)_{-}: T_{x} X \times T_{x} X \times\left(E_{x} \cap E_{0}\right) \rightarrow E_{x}$ is the curvature tensor field. It does not in general define a tensor field on $X$ in the usual sense of $x \mapsto R_{x}$ being a section of a vector bundle over $X$, because of lack of linearity in the $e$-slot. But if the connection on $E_{0}$ is homogeneous, then the maps $R_{x}(u, v)_{-}: E_{x}-0 \rightarrow E_{x}$ are smooth and homogeneous. If the connection is linear, then these maps are continuous linear, and $x \mapsto R_{x}$ defines a smooth section of $L^{3}(T X, T X, E ; E)$ $\rightarrow X$, which is the usual tensor field on $X$ (because $\partial_{2} \omega(x, a)(c) \lambda$ $=\omega(x, c) \lambda$ and $\left.\partial_{1} \omega(x, a)(\lambda) \mu=\Gamma^{\prime}(x)(\lambda)(a, \mu)\right)$.

REMARK. The structural equation and the definition of $D$ yield the following equation for the curvature tensor field of a general nonlinear connection:

$$
R(u, v) e=-D\left[u^{H}, v^{H}\right]_{e} \quad \text { for all } e \in E_{0},
$$

where $u, v$ are vector fields on $X$. (It is due to Dombrowski [2, p. 78 in the linear case.)

\section{REFERENCES}

1. W. Barthel, Nichtlineare Zusammenhänge und deren Holonomiegruppe, J. Reine Angew. Math. 212 (1963), 120-149.

2. P. Dombrowski, Geometry of the tangent bundle, J. Reine Angew. Math. 210 (1962), 73-88.

3. S. Lang, Introduction to differentiable manifolds, Interscience, New York, 1962.

4. S. Sasaki, On the differential geometry of tangent bundles of Riemannian manifolds, Tôhoku Math. J. 10 (1958), 338-354.

5. J. Vilms, Connections on tangent bundles, J. Differential Geometry 1 (1967), 235-243.

Purdue University 